\title{
Pupilometria Dinâmica para Avaliação do Reflexo Foto Motor na Detecção da Neuropatia Autonômica Diabética e Relação Glicêmica
}

\author{
Beatriz L. Bonafini ${ }^{1}$, Giselle L. F. Ronque ${ }^{2}$, Lucas F. de Oliveira ${ }^{1}$ \\ ${ }^{1}$ Departamento de Informática - Universidade Federal do Paraná (UFPR) \\ ${ }^{2}$ Departamento de Engenharia Elétrica - Universidade Federal do Paraná (UFPR) \\ CEP 82.590-300 - Curitiba, PR - Brasil \\ beatrizbonafini@gmail.com, giferrari25@gmail.com, lferrari@ufpr.br
}

\begin{abstract}
Autonomic Neuropathy is one of the complications of diabetes because it causes damage to the nervous system and one way to access it is through the analysis of the pupillary reaction. This study aimed to perform dynamic pupillometry in patients with diabetes. A video acquisition system was developed to record pupil movement exposed to light stimuli, being applied to 45 volunteers at SEMPR-HC at UFPR. 90 pupillometry videos were obtained. CNNs YoloV2 and RetinaNet were applied to the images evaluating the detection of the iris and pupil. RetinaNet achieved better performance for IoU greater than 90\%. The differences between the groups with and without neuropathy appeared in pupillary maximum dilation, plateau, and re-dilation velocity.
\end{abstract}

Resumo. A Neuropatia Autonômica é uma das complicações da diabetes por ocasionar danos ao sistema nervoso e uma forma de acessá-lo é por meio da análise da reação pupilar. Este estudo teve como objetivo realizar pupilometria dinâmica em pacientes com diabetes. Foi desenvolvido um sistema de aquisição de vídeo que registra o movimento pupilar exposta à estímulos luminosos, sendo aplicado em 45 voluntários no SEMPR-HC da UFPR. 90 vídeos de pupilometria foram obtidos. As CNNs YoloV2 e RetinaNet foram aplicadas as imagens avaliando a detecção da íris e pupila. A RetinaNet obteve melhor performance para IoU de $90 \%$. As diferenças entre os grupos com e sem neuropatia apareceram na máxima dilatação pupilar, plateau e velocidade de re-dilatação.

\section{Introdução}

O Diabetes Mellitus (DM) é uma doença caracterizada pelo distúrbio no metabolismo energético do indivíduo. Ocorre quando a glicose não é totalmente absorvida pelas células devido a ineficiência da produção do hormônio insulina [American Diabetes Association et al. 2010]. Neste contexto, indivíduos com DM, necessitam realizar o controle glicêmico por meio de auto medição, para que os níveis de glicose não tornem-se inadequados e prejudiciais ao funcionamento do corpo.

Ao longo prazo, a DM associa-se a doenças como a Neuropatia Autonômica Diabética (NAD), ocasionando a disfunção do Sistema Nervoso Autônomo (SNA), acometendo as fibras nervosas de todas as regiões do corpo. Uma das formas de manifestação da NAD é a disfunção na motricidade pupilar, que tem por consequência a 
falta de adaptação da visão no escuro, sugerindo distúrbios simpáticos e parassimpáticos [Foss-Freitas et al. 2008]. O diagnóstico precoce e o tratamento das neuropatias são importantes, pois quando detectadas pode-se amenizar os sintomas, reduzir sequelas e melhorar a qualidade de vida do paciente.

A avaliação do tamanho e simetria da pupila e da reação pupilar são imprescindíveis para o exame neurológico, pois a reação fraca ou ausente a luz podem indicar lesões cerebrais [Meeker et al. 2005]. A Pupilometria Dinâmica (PD) é uma técnica que consiste em analisar o resposta da pupila exposta a estímulos externos. Pesquisas como as de [Fotiou et al. 2000, Ferrari et al. 2010], envolvem a PD como forma de avaliação da reação pupilar sugerindo o uso de métricas que fornecem uma relação temporal do raio da pupila versus o tempo de sua constrição e dilatação.

No contexto da avaliação da PD, os métodos computacionais podem aliar-se a ela visto que técnicas de processamento de imagens e reconhecimento de padrões podem ser utilizadas no contexto da extração das características e a segmentação da pupila. Os dados obtidos então, devem servir de parâmetros para o cálculo das métricas pupilométricas e para avaliação dos padrões de normalidade no comportamento dinâmico pupilar.

Este trabalho teve como objetivo avaliar a resposta temporal da pupila de pacientes com Neuropatia Autonômica Cardiovascular (NAC) exposta a estímulos luminosos utilizando-se da PD, verificando se há correlação entre o comportamento analisado a glicemia e a NAC. As duas principais hipóteses que nortearam este trabalho foram: (1) Pacientes com DM do tipo 1 e NAC possuem reação pupilar distinta e lentificada comparada a pacientes com DM do tipo 1 em sem NAC; e (2) o valor da glicemia registrado no pacientes é capaz de influenciar o reflexo pupilar.

\section{Metodologia}

\subsection{Construção dos óculos pupilômetro}

Os componentes utilizados para a construção dos óculos pupilômetro foram: um esqueleto de óculos de realidade virtual, uma Raspberry Pi NoIR Camera V2 de 8MP e 1080p, um circuito em placa fenolite com 3 LEDs de luz infravermelha e 3 de luz branca de $5 \mathrm{~mm}$ de diâmetro cada, conectores para tensão de 5V, uma placa Raspberry Pi 3 para o controle do sistema, um banco de energia, um pen-drive de 16 GB e um ponto de acesso de rede Wi-Fi.

Para fins de visualização e organização dos arquivos durante o armazenamento das imagens, foi implementado um aplicativo acessível pela rede. O sistema armazena os dados do paciente feitos durante a coleta. A Figura 1 ilustra o funcionamento do pupilômetro. Ao ligar o dispositivo, os serviços são inicializados pelo sistema operacional Raspbian. E ao acessar o IP pelo navegador do smartphone é possível ter acesso ao aplicativo.

\subsection{Aquisição de dados em pacientes voluntários}

Após a aprovação do comitê de ética, os voluntários que preencheram os critérios de inclusão foram convidados a assinar o Termo de Consentimento Livre e Esclarecido e posteriormente realizar a avaliação de rotina, coleta dos dados clínicos, avaliação médica e realização da PD. Os critérios de inclusão para este estudo foram o diagnóstico de DM 




Figura 1. Esquema de funcionamento do pupilômetro

tipo 1 com duração de pelo menos 5 anos e idade entre 18 e 50 anos. Os critérios de exclusão foram pacientes que já possuíam doenças oftalmológicas e que tiveram intervenção cirúrgica ocular prévia.

A aquisição da PD ocorreu no Serviço de Endocrinologia do Hospital de Clínicas da UFPR no período de agosto a dezembro de 2019. Era solicitado ao voluntário que realizasse o teste de glicemia utilizando um glicosímetro, sendo registrada na momento do experimento da PD. Então, o pupilômetro era colocado no paciente, de modo que a câmera de infravermelho pudesse capturar imagens do olho direito. Em seguida todas as luzes do ambiente eram apagadas e o paciente permanecia 2 minutos no escuro, de modo que a pupila atingisse dilatação máxima. Em seguida era aplicado um flash com duração de $10 \mathrm{~ms}$ próximo ao olho do paciente, estimulando a contração da pupila, e a gravação do comportamento pupilar durante $5 \mathrm{~s}$ após o estímulo. Ao longo deste procedimento, as imagens eram armazenadas em memória para análise e processamento dos dados adquiridos. A câmera foi posicionada a aproximadamente $3,5 \mathrm{~cm}$ de distância do olho do paciente. O experimento foi aplicado de duas a três vezes no mesmo paciente. 49 pacientes participaram deste experimento, sendo que 4 foram excluídos por falhas ocorridas durante a aquisição da pupilometria, restando vídeos de 45 indivíduos para análise.

\subsection{Detecção das Regiões de Interesse e Extração das Métricas Pupilométricas}

Foram analisados 90 vídeos totalizando 13500 imagens (30 frames $/ \mathrm{s}$ ) da região do olho, com resolução de $640 \times 480$ pixels em escala de cinza. Foi realizado o processo de marcação manual com o labelImg, delimitando as fronteiras correspondentes à íris e à pupila. Devido ao tamanho da base adquirida, a marcação foi realizada em quadros intercalados em progressão de 3 imagens. Dos 150 quadros de uma captura 50 foram marcados manualmente e utilizados, resultando ao final 4264 imagens. As imagens foram utilizadas no treinamento das CNNs Yolov2 [Redmon and Farhadi 2016] (proposta por [Lucio et al. 2019]) e RetinaNet [Lin et al. 2017] para a detecção das regiões da pupila e da íris. A metodologia de validação cruzada leave-one-patient-out foi utilizada neste trabalho.

Da extração do maior diâmetro (em pixels) da íris e da pupila, foram extraídas as métricas: Amplitude do Reflexo (AMPL), Latência para Máxima Constrição (LMC), Latência para Máxima Dilatação (LMD), Latência para o Plateau (LP), Razão Pupila-Íris em Máxima Dilatação (RMD), Razão Pupila-Íris em Máxima Constrição (RMC), Razão 
Pupila-Íris em Plateau (RP) e Velocidade Média de Dilatação (VD).

O teste de normalidade de Shapiro Wilk foi aplicado aos dados e a comparação entre eles se deu pelas médias obtidas, aplicando-se o teste t e ANOVA (paramétricos), Mann Whitney e Kruskall Wallis (não paramétricos).

\section{Resultados e Discussão}

$\mathrm{Na}$ avaliação da detecção das $C N N s$ a RetinaNet obteve melhor performance para um IoU acima de $90 \%$ para a marcação manual de um especialista. Isso significa que a rede detectou mais casos no limiar de área dos bounding boxes (BB) detectados e marcados, quando a área correspondia a 90\% de intersecção entre elas. Assim ela foi aplicada para a detecção dos dados da marcação manual da união entre dois especialistas. A Figura 2 apresenta os limiares de detecção os níveis de IoU de 75\% a 95\%. Os BB em cor verde representam as marcações feitas por um dos especialistas e o vermelho detectado pela rede RetinaNet.

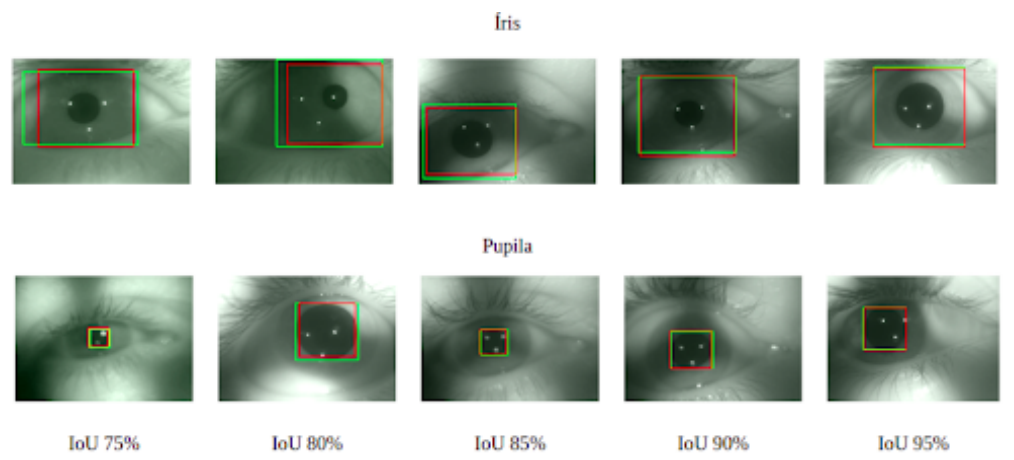

Figura 2. Imagens da PD com os bounding boxes com limiares de loU de $75 \%$ a $95 \%$

De acordo os dados clínicos obtidos pelos médicos (endocrinologistas e oftalmologista) e fisiologistas que participaram desse estudo, dos 45 pacientes analisados, 23 apresentaram NAC precoce ou definitiva, enquanto que 20 indicaram a ausência da doença. Dois pacientes foram excluídos da análise da NAC, sendo um por ter NAC de envolvimento severo e outro por apresentar fibrose cística.

Para o cálculo das métricas pupilométricas era considerado o maior diâmetro pupilar encontrado na detecção obtida. Após esta etapa, a primeira relação feita com os dados foi a verificação da relação dos níveis glicêmicos com a resposta pupilar, no qual os três níveis, sendo eles: hipo, eugli e hiperglicemia, foram considerados. Maior parte dos pacientes se encontravam em estado euglicêmico, tornando a maior o número de amostras em estado normalizado de glicemia. Assim, os dados não apresentaram diferenças estatísticas o suficiente para distinguir o estado em que o paciente se encontrava.

Também foi feita uma comparação da PD com os dados relacionados a idade dos pacientes, uma vez que o intervalo entre elas era considerável e que quanto mais tempo de doença mais possíveis danos acometem os pacientes. Nesta análise foi possível perceber que houveram diferenças significativas relacionadas as métrica de Velocidade de Re-Dilatação. Visto que a diferença apareceu para idade, a análise foi aplicada a para a 
duração do DM. Não foi possível perceber diferenças da PD entre pacientes com tempo de duração da DM distintos.

Por fim, foram aplicados os testes relacionados a presença precoce ou definitiva da NAC e a PD. A Tabela 1 apresenta os dados clínicos obtidos e os dados da PD.

\begin{tabular}{|c|c|c|c|}
\hline Parâmetros & Total & Com NAC & Sem NAC \\
\hline Número de indivíduos & 43 & 20 & 23 \\
\hline Idade (anos) & $31,09 \pm 08,26$ & $28,75 \pm 09,06$ & $33,22 \pm 07,24$ \\
\hline Duração do DM (anos) & $16,68 \pm 08,38$ & $16,30 \pm 10,07$ & $16,87 \pm 06,99$ \\
\hline HbA1c (\%) & $09,18 \pm 02,06$ & $09,19 \pm 02,04$ & $09,17 \pm 02,19$ \\
\hline $\mathrm{N}^{\circ}$ amostras PD & 86 & 40 & 46 \\
\hline AMPL (\%) & $00,17 \pm 00,06$ & $00,18 \pm 00,07$ & $00,16 \pm 00,03$ \\
\hline LMC (s) & $00,33 \pm 00,28$ & $00,34 \pm 00,34$ & $00,32 \pm 00,32$ \\
\hline LMD (s) & $03,77 \pm 00,96$ & $03,55 \pm 01,12$ & $03,96 \pm 00,76$ \\
\hline LP (s) & $02,10 \pm 01,00$ & $02,25 \pm 01,06$ & $01,97 \pm 00,93$ \\
\hline RMC (\%) & $00,35 \pm 00,06$ & $00,36 \pm 00,06$ & $00,33 \pm 00,06$ \\
\hline RMD (\%) & $00,52 \pm 00,09$ & $00,54 \pm 00,11$ & $00,50 \pm 00,07$ \\
\hline $\mathbf{R P}(\%)$ & $00,49 \pm 00,09$ & $00,51 \pm 00,11$ & $00,47 \pm 00,07$ \\
\hline VD $(\% / s)$ & $00,06 \pm 00,07$ & $00,07 \pm 00,10$ & $00,05 \pm 00,01$ \\
\hline
\end{tabular}

Tabela 1. Características clínicas e métricas da PD da amostra dos indivíduos analisados na avaliação da NAC

Ao aplicar os testes aos dados as diferenças apareceram nas métricas RMD, RP e VD. É possível observar que em média o valor de RMD para pacientes com NAC é maior, mostrando que em média a dilatação da pupila dos pacientes com NAC foi maior em relação aos sem NAC, entretanto o desvio padrão é maior, evidenciando que neste grupo essa métrica teve maior variação entre os valores analisados. Vê-se também que apesar da dilatação ser maior, o raio em constrição (métrica RMC) também é maior, evidenciando que dilatou mais, porém, em média, constringiu menos. $\mathrm{O}$ mesmo fato acontece para a métrica RP, onde a média da dilatação em Plateau é maior em pessoas com NAC do que sem NAC, porém o desvio padrão neste grupo também é maior.

A métrica VD mostra o quão rápido a pupila foi da sua máxima constrição a sua máxima dilatação após o estímulo, indicando o valor da medida da pupila pelo tempo, mostrando a velocidade de reação da recuperação do reflexo pupilar. Esta métrica apesar de se mostrar maior em média em pacientes com NAC, acaba refletindo apenas a velocidade de recuperação do estado de constrição para o estado de recuperação/re-dilatação da pupila. A recuperação deste estado pode ser mais rápida, no entanto não expressa a capacidade de constrição e dilatação da pupila - uma vez que o estado de dilatação e constrição são coordenados pelas vias do SNA simpático e parassimpático - sistemas então avaliados para a NAC.

No geral, observando as métricas para os indivíduos com e sem NAC, é possível perceber que os pacientes com NAC tiveram em média uma velocidade com maior desvio padrão em relação aos pacientes sem NAC. Tal fato mostra que o grupo desses pacientes em média tiveram também, uma resposta alterada para a recuperação do tamanho da pupila. 


\section{Considerações Finais}

O presente trabalho teve como objetivo avaliar a resposta da reação pupilar em pacientes com DM do tipo 1. A escolha da avaliação de pacientes com tipo 1 dá pelo fato da doença aparecer mais cedo nos pacientes e assim não ter maiores fatores de confusão com outras comorbidades. Para essa análise se fez necessária a construção de um dispositivo pupilômetro capaz de ser utilizado em voluntários com DM do tipo 1. Após a coleta dos dados clínicos e da PD nos pacientes, foram aplicados os métodos de detecção com CNNs para obter-se o diâmetro da pupila e da íris, que ao final serviram como parâmetros no cálculos das métricas pupilométricas. Alguns pontos para a melhoria na avaliação da PD foram identificados, como: o aumento no intervalo de gravação da resposta pupilar momentos antes e após o estímulo luminoso; marcação e estimação em milímetros do raio da pupila e da íris dos indivíduos; ajuste de foco mais preciso e/ou automático da câmera para que as imagens fiquem mais nítidas; a utilização de uma arquitetura de rede neural capaz de segmentar ao invés de apenas detectar a região da pupila e da íris; e a criação de uma base de PD em pacientes controle. Assim, busca-se futuramente aplicar os métodos nesta pesquisa utilizados para a avaliação da NAC em mais pacientes e também em indivíduos com outras doenças que acometem o SNA, e que de alguma forma influencie as respostas autônomas da pupila.

\section{Referências}

American Diabetes Association et al. (2010). Diagnosis and classification of diabetes mellitus. Diabetes care, 33(1):62-69.

Ferrari, G. L., Marques, J. L., Gandhi, R. A., Rajiv, S. R., Schneider, F. K., Tesfaye, S., and Gamba, H. R. (2010). Using dynamic pupillometry as a simple screening tool to detect autonomic neuropathy in patients with diabetes: a pilot study. Biomedical Engineering Online, 9(1):26.

Foss-Freitas, M., Junior, W., and Foss, M. (2008). Autonomic neuropathy: a high risk complication for type 1 diabetes mellitus. Arquivos Brasileiros de Endocrinologia e Metabologia, 52(2):398-406.

Fotiou, F., Fountoulakis, K., Goulas, A., Alexopoulos, L., and Palikaras, A. (2000). Automated standardized pupillometry with optical method for purposes of clinical practice and research. Clinical Physiology, 20(5):336-347.

Lin, T.-Y., Goyal, P., Girshick, R., He, K., and Dollár, P. (2017). Focal loss for dense object detection. In Proceedings of the IEEE international conference on computer vision, pages 2980-2988.

Lucio, D. R., Laroca, R., Zanlorensi, L. A., Moreira, G., and Menotti, D. (2019). Simultaneous iris and periocular region detection using coarse annotations. In 2019 32nd SIBGRAPI Conference on Graphics, Patterns and Images (SIBGRAPI), pages 178185. IEEE.

Meeker, M., Du, R., Bacchetti, P., Privitera, C. M., et al. (2005). Pupil examination: validity and clinical utility of an automated pupillometer. Journal of Neuroscience Nursing, 37(1):34.

Redmon, J. and Farhadi, A. (2016). Yolo9000: Better, faster, stronger. arXiv preprint arXiv: 1612.08242 . 